\title{
Ciencia para el matrimonio y la familia: Propuesta de un modelo basado en una filosofía realista
}

\author{
Science for marriage and family: \\ Proposal of a model based on a realistic philosophy
}

Gonzalo Flores-Castro Lingán

Seminario Arquidiocesano San Jerónimo, Arequipa, Perú

Email: gonzalofcl_1@hotmail.com

Recibido: 01-09-18

Aceptado: 20-11-18

\section{Resumen}

¿Puede integrarse la filosofía clásica del hombre en la ciencia humana? Los modelos al uso no permiten hacer una integración sin que aparezcan las categorías propias de la antropología y la ética filosófica como un complemento de la teoría, o como una hermenéutica de los resultados. Esto es especialmente grave para quienes estudian el matrimonio y la familia, pues utilizan bastantes datos de ciencias humanas interpretados a la luz de una filosofía. En este artículo mostramos, a partir de un modelo basado en una teoría de sistemas, que la filosofía del hombre (antropología y ética) puede ser integrada para hacer ciencia, creando criterios para considerar las realidades humanas de la libertad, las virtudes y los vicios (aprendizaje) en un contexto de relaciones humanas.

Palabras clave: Aristóteles, teoría de la acción, antropología filosófica, ciencias humanas, familia.

\begin{abstract}
Can the classic philosophy of man be integrated into human science? The models that are being used do not allow an integration of the categories of philosophical anthropology and ethics without appearing as an addition to the theory, or as a hermeneutics of results. This is especially serious for those who study marriage and
\end{abstract}


family, because they use a considerable number of human science data interpreted in the light of a philosophical perspective. In this article we expose that the philosophy of man (anthropology and ethics) can be integrated to make science, creating criteria to consider the human reality of freedom, as well as virtues and vices (learning) in a context of human relationships, from a model based on systems theory.

Key words: Aristotle, Action Theory, Philosophical Anthropology, Human Sciences, Family.

\section{Introducción}

Es de notar la falta de modelos científicos serios que tomen sus principios, cual ciencia subalternada, en una filosofía clásica-realista ${ }^{1}$. Estoocurre de manera especial en las ciencias sociales, muchas de las cuales utilizan modelos que no permiten integrar temas centrales de una antropología y una ética clásicas, como la libertad, las virtudes, la intimidad, el otro, etc. Ejemplos de ello podemos encontrarlos en economía (Argandoña, 2006), management (Alcázar, 2015), psicología (Ripperger, 2013) o sociología (Lucas Marín, 2011)2. Todo esto resulta especialmente relevante para quienes estudian una parcela muy importante de la realidad humana: el matrimonio y la familia, pues fundamentan sus investigaciones en modelos de distintas ciencias sociales. Esto conlleva que los resultados de sus estudios no siempre permitan una interpretación clásica-realista, sino que se perciban dichas categorías como un 'añadido' accidental a los mismos resultados.

La filosofía clásica-realista, es decir, aquella que sostiene la existencia de un mundo real independiente de nuestro pensamiento, inteligible para las personas - tanto individual como colectivamente-, con un orden que descubrimos y al cual nos debemos (Cfr. Wild, 1948; y Feser, 2014), tiene suma importancia para las ciencias del matrimonio y la familia por diferentes razones. No es nuestro objetivo exponer ni profundizar dichas razones, pero podemos mencionar al menos dos. En primer lugar, se sostiene que el ser humano es social por naturaleza, lo cual implica que la familia no es un constructo humano, sino una realidad natural que fluye de un orden que las personas descubren. Esto fundamenta una posible ciencia de la familia y no un simple 'arte' de la familia (una construcción social, idea moderna asociada al

1 Con filosofía clásica-realista me refiero a la tradición filosófica que tiene su origen en Platón y Aristóteles, así como en sus comentadores escolásticos y sus expositores contemporáneos.

2 Lo cual no quiere decir que no existan modelos que intenten hacer dicha labor, por ejemplo, en psicología, algunas corrientes humanistas basadas en la philosophia perennis - Rudolf Allers - o desde el existencialismo y la fenomenología - Viktor Frankl - toman categorías que integran una antropología filosófica clásica (Wenceslao, 2016). Sin embargo, una crítica que puede hacerse a dichos intentos es que, en muchos de los casos, se acercan demasiado a la filosofía, siendo más cercanos a la contemplación teórica que a una teoría orientada a la praxis (explicación y predicción, dominio de la realidad) propia de los modelos científicos. 
individualismo liberal). En segundo lugar, para la filosofía clásica-realista, el ser humano es capaz de virtudes, es decir, de un crecimiento y una mejora libre pero con un orden que también descubre, lo que permite la mejora en su realidad social (matrimonial y familiar).

Dado este panorama, me parece necesario proponer un modelo teórico que integre la filosofía clásica, tomando en cuenta los diversos planos de la realidad, respetando sus diferencias ontológicas (materia inerte; vida vegetativa, vida animal y vida humana). Para ello, nos serviremos de la propuesta del profesor Pérez López (1991, 1996), que intenta ser, según su propia terminología, una lógica material (Polo, 1998), es decir, un estudio de las condiciones y las propiedades lógicas que se requieren, en general, para obtener una conclusión científicamente verdadera (Millán-Puelles, 2001). Pérez López integra en su modelo aquellas categorías de la realidad descubiertas y sistematizadas por la filosofía clásica, en especial la de cuño aristotélico-tomista (Pérez López, 1991; Rosanas, 2006; Melé, Chinchilla y LópezJurado, 2018). Dichas categorías permiten conceptualizar las variables mínimas necesarias para hacer ciencia del hombre, tomándolas como supuestos explícitos a la hora de realizar interpretaciones y juicios acerca de los resultados de otras ciencias humanas o en el planteamiento inicial de un programa de investigación científica. De tomarse en cuenta solo algunas variables, los modelos científicos podrían considerarse, en términos perezlopianos, abstracciones incompletas, es decir, modelos que no proporcionan un conocimiento científico total de la realidad humana.

Cabe resaltar que dicho modelo para la consultoría y el análisis de situaciones familiares es aplicado con éxito en distintas instituciones de habla hispana, por ejemplo, la Universidad de Navarra, en España ${ }^{3}$; la Universidad Austral, en Argentina ${ }^{4}$; o el PAD Escuela de Dirección de la Universidad de Piura, en Perú5. Así también es aplicado en publicaciones de difusión (Cfr. Chinchilla \& León, 2005; Chinchilla \& Moragas, 2007; Moragas Freixa, 2014) y científicas (Chinchilla, Moragas Freixa \& Kim, 2012; Seperak Viera, 2016). Sin embargo, se observa la falta de exposición en ambos tipos de publicación del modelo perezlopiano y sus consecuencias epistemológicas. Por tanto, este trabajo tiene por objetivo ofrecer una introducción al modelo de Pérez López, a manera de complemento teórico de estudios empíricos en ciencias de la familia, asunto aún no tratado en publicaciones de esta naturaleza y del que se ocupan otras ciencias sociales, como la psicología

3 Se puede acceder a su Instituto de Ciencias para la Familia en https://www.unav.edu/matrimonioyfamilia/ y a su Centro Internacional para el Trabajo y la Familia en https://www.iese.edu/es/claustro-investigacion/ centros-investigacion/icwf/

4 Pueden verse sus distintas licenciaturas en Familia en http://www.austral.edu.ar/familia/

5 Puede verse su programa Persona y Familia en https://pad.edu/programas/programas-de-extension/ programa-persona-y-familia/ 
(García Parra, 2004), la economía (Argandoña, 2007), el management (Melé, Chinchilla \& López-Jurado, 2018) o la educación (Flores-Castro, 2018). En el ínterin, haremos explícita la integración de la filosofía clásica con el modelo propuesto, de manera que aporte a su hermenéutica y dé un marco referencial para la crítica y la interpretación de otros modelos menos completos.

\section{Antropología analítica: Teoría de la acción}

Empezaré esta exposición haciendo explícita la antropología filosófica que sustenta mi argumentación, y para ello utilizaré las conclusiones alcanzadas por una escuela particular: el realismo clásico presentado por Aristóteles y Tomás de Aquino. Se considera que el lector tiene cierto manejo de los conceptos y las categorías de dicha escuela, aunque se irán introduciendo algunas en la exposición del modelo ${ }^{6}$. Por otra parte, Pérez López construye su modelo —al cual llama antropología analítica - usando como herramienta la teoría de sistemas, cuyos conceptos iré exponiendo.

El concepto de sistema "ha invadido todos los campos de la ciencia y penetrado en el pensamiento y el habla populares y en los medios de comunicación de masas" (Von Bertalanffy, 2004, p. 1). Es cada vez más utilizado y proporciona categorías que superan la filosofía mecanicista que sostiene a muchos modelos científicos ${ }^{7}$. Como afirma Agazzi (2011), haciendo referencia a las ciencias biológicas:

Ya el viejo vitalismo decimonónico, de hecho, había subrayado no sin eficacia ciertos límites e insuficiencias de las pretensiones mecanicistas en la interpretación de los seres vivos, pero sin luego llegar a proponer una convincente alternativa a las mismas, porque la había buscado en ciertos principios sustanciales (como la "fuerza vital" o "entelequia") en lugar de una elaboración exacta de principios categoriales distintos de los mecánicos. La teoría de sistemas hace justo esto y he aquí uno de los aspectos centrales de su interés filosófico. Esta expresa, de hecho, un "modo de pensar" de tipo holístico, abierto a las consideraciones de carácter estructural, centrado en la noción de interdependencia y de acción simultánea de partes que concurren a la realización de un objetivo común, recuperando, así pues, en cierta forma la noción misma de finalidad e incluso la de "valor". Se trata [...] de conceptos que ya hacía mucho tiempo constituían contenidos intelectuales de la filosofía provistos de muy escasa repercusión en la ciencia, hasta el punto que dentro de la misma filosofía se había ido abriendo camino la convicción de que no eran utilizables como instrumento de conocimiento, sino de que, a lo sumo, podrían servir de criterios unificadores de la experiencia a título analógico o simbólico (p. 218).

6 Se encuentra una excelente exposición de la antropología clásica en Daniel Q. McInerny (2016) o en Gianfranco Basti (2003), y de la ética clásica en Ralph McInerny (1997).

7 Para un estudio avanzado de la teoría de sistemas - apta incluso para biología pero no para humanos - se sugiere la lectura de Von Bertalanffy (2004) y del mismo Pérez López (1991), la cual es apta para realidades humanas. 
Teniendo esto en cuenta, empiezo la construcción del modelo ${ }^{8}$. Un sistema es una entidad que "soporta" una particular relación entre una serie de inputs o entradas - estímulos o acciones que recibe del entorno- y una serie de outputs o salidas - "respuestas" o acciones del sistema hacia el entorno- (Pérez López, 1974b), utilizando categorías abstractas y formales, lo que permite prescindir de la naturaleza real de las entidades que se estudian (Pérez López, 1974a) ${ }^{9}$. Así, tomando en cuenta las entidades estudiadas como sistemas - a los cuales llamaremos indistintamente agentes - , podemos intentar explicar la acción de un sistema de la siguiente manera:

Figura 1.

Modelo mínimo de sistema

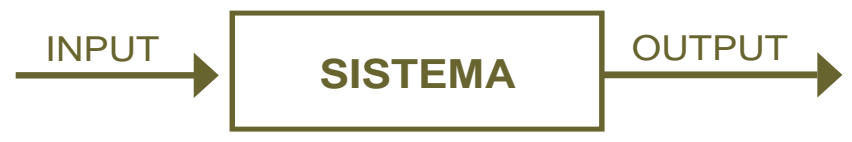

Fuente y elaboración propia.

Las acciones o el comportamiento del sistema (el output) se pueden explicar y predecir si conocemos los inputs o entradas al sistema y conociendo el estado de sistema que tenemos frente a nosotros. Si el sistema trata acerca de entes inertes, las acciones pueden ser explicadas por la medición del input (por ejemplo, la cantidad de fuerza aplicada) y el estado del sistema (su peso o su masa), que a su vez es cuantificable. Por ejemplo, a una pelota que tiene una masa igual a 0.45 kilogramos (estado del sistema) se le administra una fuerza de 90 newtons (input) y si se aplica la segunda ley de Newton, podemos concluir que la pelota presentará una aceleración de 200 metros sobre segundo al cuadrado. En este tipo de ejercicios podemos ignorar la naturaleza del sistema mismo, pues solo nos interesan aquellos aspectos cuantificables (podríamos decir que partimos del supuesto filosófico de la materia como res extensa $)^{10}$. A este tipo de sistemas Pérez López (1974a) los llama sistemas

8 Nuestra manera de proceder es distinta a la de Pérez López, pero permite comprender la integración de categorías filosóficas en el modelo científico.

9 Lo que hace posible que este modelo sea compatible con diversas corrientes filosóficas realistas, por ejemplo, el personalismo de Karol Wojtyla. A su vez, no descarto la compatibilidad con otras corrientes filosóficas. 1o Un excelente ejemplo genial que usa Genta (2009) puede ilustrar mejor la cuestión: «Seguiremos [...] un problema corriente en los manuales de física y de ciencias naturales: "Un elefante se desliza por una pendiente herbosa". El estudiante avisado sabe que no es necesario ocuparse demasiado de esto; está puesto allí solo para dar la impresión de realismo. Lee más adelante: "La masa del elefante es de dos toneladas". Ahora se comienza a hablar seriamente: el elefante desaparece del problema y lo sustituye una masa de dos toneladas. ¿Qué cosa son estas dos toneladas, verdadero sujeto del problema? Se refiere a una propiedad que descubrimos vagamente como "peso" y se manifiesta en una región particular del mundo externo... No importa a qué cosa se refieren "dos toneladas"; ¿qué cosa es? ¿Cómo han entrado realmente en nuestra experiencia en modo tan preciso? "Dos toneladas" es cuanto indica el cuadrante cuando se pone al elefante sobre la balanza. Sigamos adelante. "La pendiente de la colina es de $60^{\circ}$ ". Ahora la pendiente desaparece del problema y es sustituida por un ángulo de $60^{\circ}$. ¿Qué cosa es un ángulo de $60^{\circ}$ ?... es la lectura de una división del transportador. Y análogamente para los otros datos del problema. El suelo mórbido y blando por donde 
estables y se caracterizan porque su regla de decisión es su materia cuantificable, es decir, el conjunto de operaciones - cualesquiera que estas puedan ser- por las que un sistema "elige" su acción; su comportamiento o su output ${ }^{11}$ viene dado por aquellos aspectos materiales que la componen (Cfr. Pérez López, 1991). Además, en términos filosóficos, dado un input, el ser inerte tenderá hacia la autoconservación (permanencia en el ser); lo que en términos de teoría de sistemas podemos traducir como la búsqueda de la situación de equilibrio original u homeostasis ${ }^{12}$.

Si aplicamos las mismas categorías a otro tipo de entes, como los animales y los humanos, la cuestión se complica más. En estos casos, el comportamiento (el output o las acciones futuras) no son totalmente explicables por el input, resultando difícil la cuantificación de dichas acciones. Por ejemplo, si una persona patea el cadáver de un gato, el cuerpo inerte de este presentará un comportamiento que es enteramente explicable mediante el input y el estado del sistema en términos cuantificables. Entonces, no existe mucha diferencia entre este ejemplo y el de la pelota en el párrafo anterior. De hecho, si repetimos la acción en diferentes momentos (tanto en el caso del cadáver del gato y el de la pelota), ceteris paribus, el comportamiento será siempre el mismo. En términos cibernéticos, no habría un cambio en la regla de decisión del sistema, siendo este tipo de sistemas considerado reglas de decisión materializadas $^{13}$. Sin embargo, si una persona patea a un gato que está vivo, el comportamiento del gato no será como en el caso anterior, podría retorcerse en el aire para poder aterrizar de pie, podría haber visto el pie que se acerca a velocidad y haber reaccionado espontáneamente aplicando un determinado plan de acción que está en su programación o en su memoria (en términos cibernéticos), o haber actuado según lo que conoce sensiblemente (sentidos externos e internos, especialmente la

se desliza el elefante es reemplazado por un coeficiente de atracción, de condición análoga a la lectura de un índice... Se trataba de encontrar el tiempo de descenso del elefante y la respuesta es dada por la indicación de las agujas de los minutos y de los segundos sobre el cuadrante de nuestro reloj» (p. 2o). Por otra parte, entender la materia como res extensa no carece de problemas, usamos ese supuesto a manera de ilustración. Para quien desee profundizar en dicho tema, recomiendo la lectura de Feser (2009) y Burtt (1925).

11 Nótese que en el caso de un ser inerte, su "elección" viene dada por aquellos factores cuantificables del sistema, a diferencia de lo que pasará en un animal o un humano. No es necesario recurrir a la noción metafísica de intencionalidad, aunque la interpretación del término lo reclama. De hacerlo así, la materia inerte también presenta un tipo de intencionalidad inorgánica, pues, aunque no es una "elección" consciente, "tenderá" a ciertos comportamientos dada su naturaleza (Cfr. Oderberg, pp. 259-279).

12 "Procesos merced de los cuales se mantiene la situación material y energética del organismo" (Pinillos, 1975, p. 16). Según Von Bertalanffy (2004): «Muchas regulaciones psicofisiológicas siguen los principios de la homeostasia. Sin embargo, hay limitaciones evidentes [...]. Podemos decir que la homeostasia es inapropiada como principio explicativo para las actividades humanas no utilitarias, que no sirven a las necesidades primarias de conservación propia ni a sus derivaciones secundarias, como ocurre con tantas manifestaciones culturales [...]. El principio de homeostasia a veces ha sido hinchado hasta el punto de hacerlo ridículo. La muerte del mártir en la pira es explicada (Freeman, 1948) por el "desplazamiento anormal" de sus procesos internos, de manera que la muerte es más "homeostatizadora" que seguir con la vida (pp. 142 y ss.); se supone que el alpinista arriesga la vida porque "el perder un estatus social apreciado puede ser más perturbador" (Stanger, 1951)» (pp. 8o-81).

13 Cabe notar que el desgaste material o el añadido de nueva materia al sistema puede implicar cambios en las acciones futuras. No se toman en consideración estas variables dado que los ejemplos solo son ilustrativos. 
estimativa animal) y actualizar su apetito elícito, sintiendo aversión y alejándose, esquivando la patada, arañando el pie que lo patea, etc., en términos de psicología clásica. Por otra parte, al intentar patear a dicho gato en el futuro, de darse cuenta este, probablemente sepa evitar el golpe, es decir, es posible que haya aprendido, que haya un cambio en sus reglas de decisión (en términos cibernéticos).

¿Qué explica que en el caso de un ser viviente ocurra tal cosa? Al parecer, el ser viviente animal es un tipo de ente que es aportante, da algo de sí que no es objeto de las ciencias empíricas o cuantificables. En palabras de Von Bertalanffy (1971), uno de los padres de la cibernética:

\begin{abstract}
No existe ninguna diferencia entre los procesos físicos y químicos que se desarrollan en un organismo vivo y los que lo hacen en el cadáver. Unos y otros obedecen a las mismas leyes físicas y químicas, eso es todo lo que se puede decir. Pero para el biólogo y para el médico existe una profunda diferencia entre lo organizado y lo que se desordena para destruirlo. ¿Cuáles son los principios de ordenamiento y de organización? ¿Qué quieren decir "salud" y "norma” en contraste con "enfermedad" y "patología"? Nada, en lo que atañe a las leyes físicas y químicas y cuando se adopta una filosofía mecanicista ${ }^{14}$. Mas sin ideas como estas y otras parecidas no existiría la ciencia ni la medicina, ni tampoco habría biología (p. 100).
\end{abstract}

Para Von Bertalanffy, el ser vivo tiene una diferencia con el cadáver, y debemos tenerla en cuenta para crear modelos que nos permitan explicar y predecir acciones aquí y en el futuro, es decir, hacer ciencia. Filosóficamente hablando, el ser vivo es un todo no explicable por sus partes físicas (el todo no es solo la suma de sus partes), y debe ser estudiado sistemáticamente y no analíticamente, como afirma Polo (2002): “[...] Un cuerpo vivo es un todo complejo cuyos elementos son todos pertinentes. Desde este punto de vista, el sistema no es tratable analíticamente". En la tradición clásica, el ser vivo se diferencia porque presenta automovimiento, es decir, algo parte de ellos (McInerny, 2016). Además, dicha tradición filosófica afirma que la diferencia entre el ser vivo y el ser inerte estriba en que el primero es capaz de operaciones inmanentes (Oderberg, 2013). Es decir, algo permanece en el ser vivo al momento de realizar una acción. Esto puede verificarse en la física moderna con el intercambio de energía que hace el ser vivo para permanecer organizado o en orden, que al parecer entra en conflicto con el segundo principio de la termodinámica, el cual indica que la entropía decrece, es decir, explica la tendencia natural de los sistemas a la desorganización y al frío (Mosterín, 2011). No todo sistema hace esto, solo los vivientes, por lo cual se deduce que deben aportar algo ellos mismos (su configuración, su información o su orden, en el caso del ADN), a lo que llamamos automovimiento, pero además que algo queda con ellos, como dice Mosterín (2011), "absorbemos materia y energía, que transformamos en nuestra propia substancia y utilizamos para nuestras propias funciones, y excretamos nuestros residuos" (p. 59), es decir, inmanencia.

14 La cursiva es mía. 
Al integrar ambas categorías filosóficas al modelo, podemos decir, en primer lugar, que este tipo de sistemas presenta un estado interno que explica y predice sus acciones. De hecho, podemos obtener diferentes tipos de sistemas que se clasifican por dichas categorías. La segunda categoría - la inmanencia-, en términos de sistemas, podemos llamarla aprendizaje, y la entenderemos como:

[...] Cualquier cambio que ocurra en los agentes [sistemas] como la consecuencia de la ejecución del proceso de interacción, siempre que dicho cambio sea significativo para la explicación de las futuras interacciones. Así pues, por "aprendizaje" entendemos aquellos cambios que ocurren en el interior de los agentes como consecuencia de la propia interacción, siempre que esos cambios influyan en cómo será la siguiente interacción. Si denominamos regla de decisión al conjunto de operaciones - cualesquiera que estas puedan ser- por las que un agente [elige su acción], el aprendizaje es el concepto que utilizaremos para recoger los cambios en las respectivas reglas de decisión, cambios que han sido provocados por la interacción (Pérez López, 1991, p. 28).

La siguiente categoría, el automovimiento (aquello que aporta el sistema), podemos incluirla en el modelo como la independencia del sistema frente al input con respecto a su output. De tener un sistema cuyos mecanismos internos - aquellos constructs que explican la acción - están determinados de tal manera que con cada input hay una modificación que siempre busca el equilibrio u homeostasis y cuyo aprendizaje, a su vez, determina la regla de decisión a seguir (es decir, hay cierta independencia frente al input pero no frente al propio estado del sistema, actual o futuro) ${ }^{15}$, estaremos frente a un sistema ultraestable (Pérez López, 1974a). Dicho sistema tendrá siempre un aprendizaje positivo, es decir, toda retroalimentación o aprendizaje -inmanencia - servirá para que el sistema llegue a su equilibrio. Este tipo de sistemas nos pueden ayudar a modelizar el comportamiento de un ser vivo (Von Bertalanffy, 2004).

¿Qué sucede en el caso del ser humano? En este punto, volvemos a lo mencionado en la introducción: no hay una genuina integración de categorías filosóficas clásicas en los modelos científicos al uso. Por ejemplo, se intenta introducir categorías filosóficas clásicas como la libertad (que es fundamento de la virtud) para explicar realidades de pareja o familia. Sin embargo, se hace como un agregado a los modelos científicos. Hay reflexiones excelentes $-\mathrm{y}$, en mi opinión, verdaderas- desde un punto de vista filosófico, por ejemplo, Melendo y Millán-Puelles (2005), hablando sobre el amor libre y el matrimonio, afirman que:

15 Volvamos al ejemplo del gato y el cadáver del gato: el comportamiento del cadáver del gato viene determinado por el input — la patada - y el estado externo o cuantificable del sistema — su peso-; en el caso del gato vivo, su comportamiento es condicionado por dichos aspectos, pero no determinado por ellos, porque depende del estado interno del sistema, es decir, está determinado por la configuración del sistema mismo - sus instintos, su programación original (ADN), etc. - o por su experiencia pasada (conocimiento experimental o información en el archivo de la memoria del sistema). 
La libertad empieza a advertirse como el inefable atributo que eleva al hombre por encima de los animales cuando caigo en la cuenta de que cada una de las acciones que realizo libremente deja una propia huella en mi propio ser, me modifica, haciéndome crecer (ser más y mejor) o si fuese el caso, des-haciéndome, disminuyendo la estatura de lo que soy. Esa modificación puede resultar muy tenue, cuando lo que pongo en juego al obrar es un aspecto periférico o menos trascendente de lo que me constituye como persona: cuando decido hacer un pequeño esfuerzo para ayudar a un amigo o me privo, por amor a quienes quiero, de un capricho insignificante. Pero también puede incidir de manera profunda en mi vida, configurándome de un modo nuevo: como sucedería [...] cuando me arrepiento honda y sinceramente de una actitud arraigada o una costumbre que ponía trabas a mi despliegue personal, y decido cambiar de vida (pp. 46-47).

Esta reflexión resulta difícil de aplicar para quienes estudian sociología o psicología, ciencias en las que se intenta utilizar categorías sin una valoración de bien o mal, es decir, aspectos éticos de la acción (Lucas Marín, 2011). Por lo que las elecciones libres - buenas o malas - terminan como un agregado o una interpretación de la data obtenida. En cambio, en el modelo de Pérez López puede integrarse el atributo libertad cuando se habla de la independencia del sistema no solo frente a los inputs, sino frente al propio estado interno del sistema: el aprendizaje condiciona fuertemente la acción del sistema, pero queda espacio para que el propio sistema decida cuál será su rumbo de acción ${ }^{16}$. Además, la realidad del hábito (sea virtud o vicio) queda intacta en la noción de aprendizaje positivo y negativo, lo que nos permitirá usar categorías funcionales para su aplicación científica.

El agente personal tiene el máximo automovimiento conocido experimentalmente, es decir, es fundamentalmente aportante, es don o dar (Sellés, 2008). Esta noción filosófica puede entenderse como un sistema libremente adaptable (freely adaptive system) (Pérez López, 1974a), en el cual el aprendizaje puede ser negativo, es decir, que no se busque su estado de equilibrio, que en el ser humano es su felicidad. En palabras de Llano (2008):

Con todas mis limitaciones, soy como un avanzadísimo ingenio cibernético: aprendo de mis aciertos y de mis errores. Cuando aprecio mis aciertos, refuerzo esa línea de conducta y la enriquezco con el registro de los nuevos casos, diversificando así las posibilidades de acción. Cuando detecto mis errores, retroalimento las posiciones de partida, atemperando las actitudes que han conducido a los diversos fallos, y potenciando aquellas que puedan neutralizarlas y sustituirlas. [...] Pero en mi actividad acontece también el aprendizaje negativo, en el que desgraciadamente ya no se registra esa versatilidad del autogobierno. Baste pensar en fenómenos como la envidia, el engreimiento o el rencor, que no me

16 En sentido estricto, la libertad humana no puede entenderse como independencia absoluta del entorno. En todo caso, el único ser completamente independiente es Dios, el Ipsum Esse Subsistens. Nosotros participamos directamente del Ser que es Dios, y por ello somos libres (Pérez López, 1992). Esto se ve claramente en la necesidad de las personas de resolver sus problemas y ser felices (Flores-Castro, 2018). 
son desconocidos. El más temible de todos ellos es el resentimiento, porque me puede paralizar durante mucho tiempo. [...] El pasado comienza a ser un factor insoportable que amenaza mi capacidad de rectificación. Incomprensible, pero de modo muy real, empiezo a hacerme daño a mí mismo. Si insistiera en esa trayectoria, sería el comienzo de la vida malograda (pp. 27-28).

Así, teniendo un sistema libremente adaptable (o simplemente sistema libre) que posee cierta independencia frente a los inputs para explicar su comportamiento, cierta independencia frentea su propioaprendizaje — sea positivo o negativo (noción de virtud o vicio) - para explicar su actuar futuro, obtenemos un modelo mínimo para explicar el comportamiento humano. Solo faltaría deducir los mecanismos internos necesarios (constructs) que hacen posible este sistema. Estos constructs los desarrolla Pérez López (1991) y son compatibles con la misma antropología y la misma ética clásica (precisamente porque presupone lo visto hasta este punto), y usan categorías abstractas como racionalidad, virtualidad, motivación potencial, motivación actual, conocimiento experimental, conocimiento abstracto, etc., los cuales no desarrollaré en este trabajo. Interesa ahora un punto fundamental en lo que respecta al modelo mismo: la persona como ser relacional, social por naturaleza.

\section{Teoría de la acción en las organizaciones}

El modelo del profesor Pérez López no termina allí, sino que aplica dichas nociones a una realidad organizacional, es decir, a las consecuencias sociológicas de su modelo (Pérez López, 1996). Este es un dato filosófico que integra Pérez López en su modelo, pues como dice Sayés (1997):

Otra dimensión inequívoca de la existencia humana es la relación con el otro. El hombre es un yo, particularmente, en relación con un tú dentro del horizonte del amor. El otro se le presenta al hombre no como pedazo del mundo material que puede instrumentalizar; sino como un ser de igual dignidad que la suya, de tal modo que le debe un respeto incondicional sin que pueda jamás reducirlo a condición de medio o instrumento de sus fines (p. 42).

O como afirma Yepes Stork (2003): "El hombre es un ser familiar precisamente porque nace y muere indefenso, sin recursos, desprotegido, niño" (p. 215). En otros términos, es la aplicación del supuesto filosófico aristotélico del hombre como animal político o social por naturaleza (Cfr. Flores-Castro, 2018). Utilizando las categorías de Pérez López, podemos modelizar dicha codependencia humana de la siguiente manera: 
Figura 2.

Modelo mínimo de interacción de sistemas libres

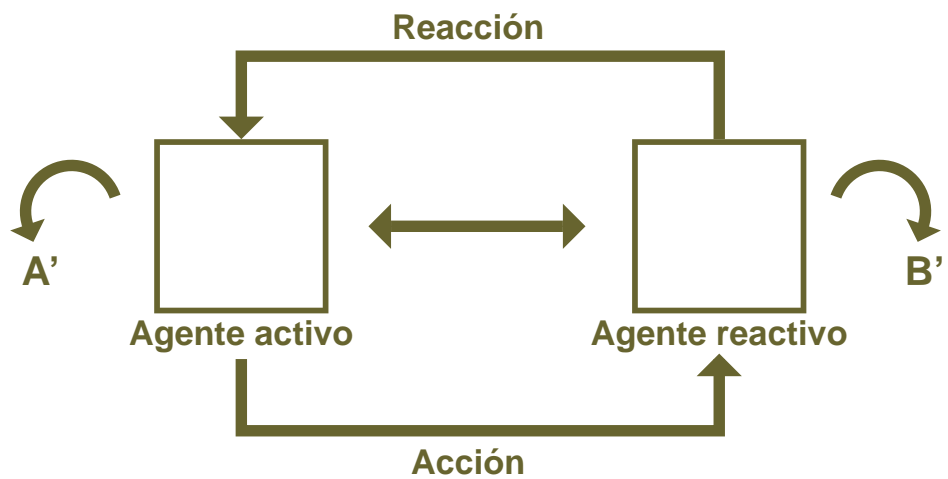

Original de Fundamentos de la dirección de empresas, de J. A. Pérez López, 1996, p. 53.

La Figura 2 muestra el modelo mínimo que dos agentes o sistemas libres presentan al interactuar entre ellos. El agente activo A tiene algún tipo de problema -que definiremos como la existencia de una situación que no es del todo agradable, o cuando se sospecha que existen situaciones más agradables que las actuales (Ariño, 2005) - que requiere de la interacción con el agente reactivo B. A realiza una acción con B para generar una reacción que resuelva su problema. Para entenderlo mejor, veamos un ejemplo: Pedro se siente solo y piensa que salir con Juana resolverá su problema; por su parte, Juana es cinéfila —dato que él conoce-, por lo que Pedro realiza una acción — invitar a Juana al cine - que Juana acepta, y se resuelve -al parecer- el problema de ambos.

Sin embargo, como hemos visto, A es un tipo de sistema que puede reajustar sus reglas de decisión según la interacción con B, es decir, aprende. Luego de la interacción, al ser B también un agente libre, puede aprender dada la interacción. Esta situación mínima nos permite deducir los siguientes resultados de la acción:

- Resultados extrínsecos: La reacción de B dada la acción de A.

- Resultados intrínsecos: El aprendizaje de A, que puede ser positivo o negativo.

- Resultados trascendentes: El aprendizaje de B, que puede ser positivo o negativo.

En el ejemplo anterior, Pedro podría estar evaluando solamente los resultados extrínsecos de su acción (la reacción que recibirá de Juana), es decir, todas aquellas variables que aseguran la consecución de la reacción. A estas variables Pérez López (1991) les llama criterio de eficacia, compuesto por tres subcriterios: 
- La evaluación de la acción en cuanto se calcula producirá la reacción necesaria para resolver el problema; a lo cual llamamos criterio de validez.

- La evaluación del propio estado interno - el estado interno del sistema A - en cuanto puede ejecutar la acción que producirá la reacción; a lo cual llamamos criterio de operacionalidad.

- La evaluación del estado interno del agente reactivo B para producir la reacción dada la acción del agente A, a lo cual llamamos criterio de instrumentalidad (u operacionalidad del agente B).

Para una sola interacción, Pedro habría evaluado bien su decisión, lo que Pérez López (1991) llama evaluación estática. Sin embargo, ¿qué pasaría si Pedro requiere de más interacciones con Juana para resolver sus problemas?; en el ejemplo que se ha proporcionado, ¿qué pasaría si requiere de más interacciones para no sentirse solo constantemente? En vista de esta situación, la evaluación de la acción de Pedro debe considerar los aprendizajes de ambos, con el riesgo de realizar una abstracción incompleta, es decir, una evaluación en la que no se consideren todos los criterios necesarios para asegurar la resolución de problemas actuales y futuros. Se requiere, por tanto, una evaluación dinámica que incluya criterios de aprendizaje (Pérez López, 1991). Así tenemos:

- La evaluación del estado interno futuro del agente activo, es decir, su operacionalidad futura. A ello le llamamos criterio de eficiencia.

- La evaluación del estado interno futuro del agente reactivo, es decir, su instrumentalidad futura. A ello le llamamos criterio de consistencia.

En el ejemplo de Pedro, para una primera interacción no parece mala idea invitar a Juana al cine. Es una acción eficaz, pero quizá no eficiente, pues de no aprender a disfrutar de las películas es posible que Pedro ya no desee repetir la interacción. Por otra parte, tal vez Juana ya no desee seguir yendo al cine con Pedro, pues se da cuenta de que él no disfruta del cine tanto como ella, así la consistencia decae. ¿Qué debe hacer Pedro? Acaso pensar en planes de contingencia, por si la acción deja de ser eficaz debido a la pérdida de eficiencia o consistencia; o pensar en un tipo de acción lo suficientemente eficaz como para asegurar la operacionalidad y la instrumentalidad futuras. Nótese que la eficacia futura depende de la eficiencia y, sobre todo, de la consistencia, ya que el agente activo tiene acceso a modificar su propia operacionalidad, no así la instrumentalidad.

Aplicando el modelo a realidades familiares, este podría ser utilizado en clave hermenéutica para otros estudios o al momento de realizar el diseño experimental de una investigación (a manera de marco referencial formal). Por ejemplo, numerosas investigaciones muestran que el ambiente familiar influye significativamente en la aparición de síntomas de depresión en los hijos. Según un artículo del Instituto para el Matrimonio y la Familia (2018): 
Estas experiencias — que incluyen desde abuso sexual, emocional, físico, negligencia física y emocional hasta problemas en el hogar, padres separados o divorciados- muestran una relación directamente proporcional con problemas de salud mental, como el consumo de drogas y alcohol, los intentos de suicidio, la depresión, entre otros.

Ahora bien, la interpretación de estos datos puede realizarse desde distintas variables, como los criterios de eficacia, eficiencia y consistencia - siendo este un modelo más general y dinámico que el utilizado-. El comportamiento de algunos padres con hijos depresivos parece haber sido eficaz para aquellos, pues resolvieron algún problema que percibían que tenían ${ }^{17}$, como, por ejemplo, la necesidad imperante de ingerir alcohol; pero olvidaron considerar el criterio de eficiencia y el de consistencia al tomar sus decisiones. La operacionalidad futura (eficiencia) puede haberse visto comprometida al consumir una cantidad de alcohol que no permita interactuar con otras personas (destruyendo la organización familia). La instrumentalidad futura (consistencia) puede haber sido amenazada al no considerarse el estado interno del otro agente (el hijo) a la hora de decidir el consumo de alcohol, destruyendo la unidad del sistema (la confianza). Toda esta hermenéutica puede guiarnos a la hora de buscar causas que expliquen diversos comportamientos y se evitarían así abstracciones incompletas. Por otra parte, sin utilizar categorías de valor o filosóficas, el modelo trae de forma implícita la posibilidad de una interpretación filosófica de corte clásico-realista.

A través de este breve análisis, simple pero completo, he integrado nociones clave de la antropología y la ética filosófica - inmanencia, libertad, hábitos- en categorías no filosóficas, de modo que pueden servir de criterios para la evaluación de teorías científicas o de integración de diversas ciencias humanas que sean tratadas en un modelo científico más amplio. Ya no se habla de libertad, es una condición de funcionamiento del modelo; ya no se habla de inmanencia, sino de aprendizaje -aquello que explica comportamientos futuros de los sistemas-; no se habla en categorías de bien o mal, sino en categorías operativas, como eficacia, eficiencia o consistencia de las interacciones de los sistemas. Todas estas categorías permiten introducir la posibilidad intrínseca del modelo a una interpretación clásica-realista sin que esta sea un agregado más al modelo.

\section{Conclusiones}

Los modelos teóricos usados en ciencias humanas no consideran realidades antropológicas y éticas propias de una filosofía realista del ser humano, como la libertad y los hábitos de la persona. Sin embargo, son realidades humanas patentes y deben estar incluidas en los modelos mismos y que no sean un mero complemento o una hermenéutica de resultados. El profesor Pérez López construye

17 Aunque este problema pueda ser un falso problema. Una introducción a problemas o necesidades reales o falsos puede verse en Ferreiro y Alcázar (2012). 
un modelo teórico que permite una integración intrínseca con la filosofía del hombre (antropología y ética), siendo una lógica material que reúne elementos relevantes de la filosofía para conceptualizarlos de manera que sean útiles y operativos para la ciencia del hombre - evitando realizar juicios de valor, tomando en cuenta otras categorías que los suponen-. Este modelo mínimo es el que resulta de la interacción de dos sistemas libres, en los cuales se toman en cuenta como variables evaluativas mínimas los criterios de eficacia, eficiencia y consistencia. De no hacerlo, el científico estará cayendo en una abstracción incompleta y no estará realizando una ciencia humana - en este caso, una ciencia del matrimonio y la familia - completa y realista.

En este documento, he expuesto y explicitado diversas nociones filosóficas propias de la antropología y la ética filosófica, analizando cómo estas participan en la construcción del modelo de Pérez López. Creemos que es una contribución al mejor entendimiento del modelo mismo y de su utilidad para las ciencias humanas en general —así como para las ciencias del matrimonio y la familia en particular-. He ejemplificado también cómo podría hacerse dicha aplicación. Cabe resaltar que la revisión del modelo presentada en este artículo no ha pretendido ser exhaustiva, pues no he tocado los constructs del modelo, es decir, aquellos mecanismos teóricos necesarios que los sistemas libres deben de presentar internamente para explicar y predecir su comportamiento futuro, así como el de la organización resultante, tema que trataré en otra oportunidad.

\section{Referencias}

Agazzi, E. (2011). La ciencia y el alma de occidente. Madrid: Tecnos.

Alcázar, M. (2015). Cómo mandar bien. Lima: Infobrax. (Obra basada en el capítulo uno de su tesis doctoral sobre Pérez López).

Argandoña, A. (2006). La ética en la economía y en las organizaciones: ¿es posible una integración efectiva? (Occasional Paper OP n. ${ }^{\circ}$ o6/17). Recuperado de la pagina web de la IESE Business School - Universidad de Navarra: www.iese. edu/research/pdfs/op-o6-17.pdf

Argandoña, A. (2007). Anthropological and ethical foundations of organization theory (Working Paper n. ${ }^{\circ}$ 707). Recuperado de la pagina web de la IESE Business School - Universidad de Navarra: https://media.iese.edu/research/ pdfs/DI-0707-E.pdf

Ariño, M. Á. (2005). Toma de decisiones y gobierno de organizaciones. Barcelona: Deusto.

Basti, G. (2003). Filosofia dell'uomo. Bologna: ESD-Edizioni Studio Domenicano.

Burtt, E. A. (1925). The Metaphysical Foundations of Modern Science. London: Kegan Paul, Trench, Trubner \& Co. Ltd. 
Chinchilla, N., \& León, C. (2005). Female Ambition. How to Reconcile Work and Family. New York: Palgrave Macmillan.

Chinchilla, N., \& Moragas, M. (2007). Dueños de nuestro destino. Cómo conciliar la vida profesional, familiar y personal. Barcelona: Ariel.

Chinchilla, N., Moragas, M. \& Kim, S. (2012). Work and family decision-making framework: A motivational perspective. (Working Paper DI-956-E). IESE Business School, University of Navarra.

Ferreiro, P., \& Alcázar, M. (2012). Gobierno de personas en la empresa (6. ${ }^{\mathrm{a}}$ ed.). Lima: Planeta.

Feser, E. (2009). The Last Superstition: A Refutation of the New Atheism. Indiana: St. Agustine's Press.

Feser, E. (2014). Scholastic Metaphysics. A contemporary introduction. Alemania: Editions Scholasticae.

Flores-Castro, G. (2018). La amistad aristotélica desde la antropología analítica de Juan Antonio Pérez López (tesis de maestría). Universidad de Piura, Piura, Perú.

García Parra, A. T. (2004). Una nueva teoría de motivación. El modelo antropológico de Juan Antonio Pérez López. Revista Puertorriqueña de Psicología, (15), 123-163.

Genta, J. B. (2009). El filósofo y los sofistas (2. ed.). Buenos Aires: Lumen.

Instituto para el Matrimonio y la Familia. (2018). Depresión y familia: una aproximación al rol de los padres. Recuperado de http://ucsp.edu.pe/imf/ investigacion/articulos/depresion-y-familia-una-aproximacion-al-rol-delos-padres/

Llano, A. (2008). La vida lograda (6. ${ }^{\mathrm{a}}$ ed.). Barcelona: Ariel.

Lucas Marín, A. (2011). Sociología: El estudio de la realidad social. Pamplona: EUNSA: Astrolabio.

McInerny, D. Q. (2016). Philosophical Psychology. Pennsylvania: The Priestly Fraternity of Saint Peter.

McInerny, R. (1997). Ethica Thomistica. Washington D. C.: The Catholic University of America Press.

Melé, D., Chinchilla M. N., \& López-Jurado, M. (2018). The "Freely Adaptive System". Application of this Cybernetic Model to an Organization Formed by Two Dynamic Human Systems. Philosophy of Management.

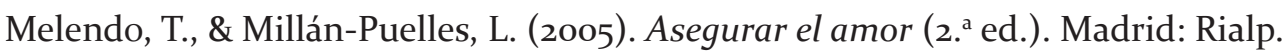
Millán-Puelles, A. (2001). Fundamentos de filosofía. Madrid: Rialp.

Moragas Freixa, M. (2014). El tiempo en un hilo. Madrid: Rialp.

Mosterín, J. (2011). La naturaleza humana. Madrid: Austral.

Oderberg, D. (20o8). Teleology: Inorganic and Organic. En A. M. González (Ed.), Contemporary Perspectives on Natural Law (pp. 259-279). Aldershot: Ashgate. 
Oderberg, D. (2013). Synthetic Life and the Bruteness of Immanent Causation. En E. Feser, Aristotle on Method and Metaphysics (pp. 206-235). Basingstoke: Palgrave Macmillan.

Pérez López, J. A. (1974a). Organizational control theory: A formal approach. (Working Paper n. ${ }^{\circ}$ ). División de Investigación del IESE.

Pérez López, J. A. (1974b). Anthropology and Sociology. (Working Paper n. ${ }^{\circ}$ 6). División de Investigación del IESE.

Pérez López, J. A. (1991). Teoría de la acción humana en las organizaciones. La acción personal. Madrid: Rialp.

Pérez López, J. A. (1992). Apuntes sobre la teoría de la acción humana en las organizaciones. Bogotá: Universidad de La Sabana. (Original de clases impartidas en el Instituto de Altos Estudios Empresariales IAE - Argentina).

Pérez López, J. A. (1996). Fundamentos de dirección de empresas. Madrid: Rialp.

Pinillos, J. L. (1975). Principios de psicología. Madrid: Alianza Editorial.

Polo, L. (1998). La figura científica de Juan Antonio Pérez López. En VV. AA., Discursos en el acto académico homenaje en memoria del profesor Juan Antonio Pérez López (p. 5). Piura: Universidad de Piura.

Polo, L. (2002). La cibernética como lógica de la vida. Studia Poliana, (4), 9-17.

Polo, L. (2007). Quién es el hombre. Un espíritu en el tiempo (6. ${ }^{\mathrm{a}}$ ed.). Madrid: Rialp.

Ripperger, C. (2013). Introduction to the Science of Mental Health. Oklahoma: Sensus Traditionis Press.

Rosanas, J. (2006). ¿Qué cosas diferentes dijo Juan Antonio Pérez López? Revista de Antiguos Alumnos del IESE, 38-42.

Sayés, J. A. (1997). Antropología y moral. De la «nueva moral» a la Veritatis splendor. Madrid: Pelícano.

Sellés, J. F. (2008). Antropología para inconformes (4. ${ }^{a}$ ed.). Madrid: Rialp. Instituto de Ciencias para la Familia.

Seperak, R. (2016). Motivos extrínsecos-intrínsecos y trascendentales en la decisión de contraer matrimonio en casados, separados y/o divorciados. Revista de Psicología, 6(1), 349-368.

Von Bertalanffy, L. (1971). Robots, hombres y mentes. Madrid: Guadarrama.

Von Bertalanffy, L. (2004). Teoría general de sistemas. México: Fondo de Cultura Económica.

Wenceslao, V. (2016). Madurez psicológica y espiritual. Madrid: Palabra.

Wild, J. (1948). Introduction to Realistic Philosophy. New York: Harper and Brothers.

Yepes Stork, R., \& Aranguren, J. (2003). Fundamentos de Antropología. Un ideal de la excelencia humana (6. ${ }^{a}$ ed.). Pamplona: EUNSA. 